\title{
La fisiología clínica en la práctica de la medicina respiratoria
}

\section{The clinical physiology in the respiratory medicine practice}

\author{
Luis Torre-Bouscoulet* \\ *Instituto de Desarrollo e Innovación en Fisiología Respiratoria (INFIRE), Ciudad de México.
}

Si he logrado ver más lejos, ha sido porque he subido a los hombros de gigantes. Isaac Newton

Los avances más importantes en la medicina respiratoria en el último siglo se los debemos, en gran medida, a los gigantes de la fisiología clínica. Sería injusto hacer una lista de ellos porque, sin duda, la omisión delataría mi ignorancia; sin embargo, la medicina respiratoria no sería la misma sin esos gigantes que, junto a la cama de los pacientes, se propusieron entender la enfermedad desde sus anormalidades funcionales. Las aportaciones de la fisiología clínica han sido la base de una práctica clínica de «avanzada» que nos ha impulsado hacia los límites del conocimiento. El conocimiento fisiológico nutre incansablemente la práctica clínica.

Ejemplos hay muchos. Sería imposible concebir las estrategias de ventilación mecánica que se utilizan en la actualidad sin la innovación derivada de la fisiología clínica. Recientemente, los fenotipos descritos para pacientes con neumonía por SARS-CoV-2 se basan en las características fisiológicas; el Tipo «L» es el fenotipo caracterizado por baja elastancia, elevada distensibilidad, baja relación V/Q y escasa reclutabilidad alveolar; mientras que el tipo «H» se caracteriza por elevada elastancia, elevada fracción cortocircuito y alta reclutabilidad alveolar. ${ }^{1}$ Los cambios fisiológicos derivados de la infección, agrupados en dichos fenotipos distinguen diferentes procesos

Correspondencia:

Dr. Luis Torre-Bouscoulet

Instituto de Desarrollo e Innovación en Fisiología

Respiratoria (INFIRE), Ciudad de México.

Correo electrónico: luistorreb@gmail.com

Trabajo recibido: 11-X-2020; aceptado: 13-X-2020.

Citar como: Torre-Bouscoulet L. La fisiología clínica en la práctica de la medicina respiratoria. Neumol Cir Torax. 2020; 79 (4): 221-223. https:// dx.doi.org/10.35366/97963 fisiopatológicos, diferentes estrategias de ventilación y diferente pronóstico.

El desarrollo tecnológico para la medición de parámetros biológicos relacionados con la respiración tiene su sustento en el conocimiento fisiológico. Las salas de operaciones y de cuidados intensivos están repletas de equipo biomédico que miden en tiempo real parámetros fisiológicos respiratorios, cardiovasculares, metabólicos, etc. El mismo escenario aplica en el seguimiento de pacientes con enfermedades respiratorias. Ha sido la fisiología clínica la base cotidiana para identificar patrones funcionales que contribuyen al correcto diagnóstico y son precisamente los más importantes en el seguimiento y en el pronóstico de los pacientes. La capacidad vital, descrita en 1856 por John Hutchinson, ${ }^{2}$ es elocuentemente vital porque es un potente predictor de mortalidad de cualquier causa, incluso en enfermedades no respiratorias. ${ }^{3}$ Son los parámetros fisiológicos $\left(\mathrm{FEV}_{1}, \mathrm{FVC}\right.$, DLCO metros caminados en seis minutos) los principales resultados en un sinnúmero de ensayos clínicos controlados. ${ }^{4}$ En el ámbito semiológico, el análisis de la disnea, por ejemplo, se basa en la comprensión de principios fisiológicos sencillos. ¿Qué autoridad intelectual podríamos reclamar ante un paciente con disnea si no comprendemos la curva longitud-tensión del diafragma, o la ecuación presiónvolumen que traduce las características de distensibilidad pulmonar? Al analizar las características funcionales de la respiración de un paciente podemos entender no sólo la enfermedad, sino el origen de sus síntomas y las posibles áreas de intervención terapéutica. Otro afortunado ejemplo de la utilidad de la fisiología respiratoria aplicada en la medicina del sueño se dio a finales de los años 70 cuando el Dr. Colin Sullivan en su natal Australia invirtió el funcionamiento de la aspiradora de su casa-asumiendo la reprimenda correspondiente- para aplicar presión positiva en la vía aérea superior, y con ello evitar el colapso faríngeo característico de la apnea obstructiva del sueño. El enorme avance terapéutico de los equipos de CPAP no se hubiera desarrollado si Sullivan no hubiera conocido los pormenores de la fisiología de la vía aérea superior. ${ }^{5}$ 
Las características fisiológicas de una persona permiten una mejor identificación de su tratamiento. Por ejemplo, si un paciente con apnea del sueño tiene una exagerada respuesta ventilatoria posapnéica, será más susceptible de desarrollar respiración periódica que su contraparte con una respuesta ventilatoria posapnéica «normal». Si un paciente con EPOC tiene hiperinflación dinámica tendrá más disnea, menor calidad de vida, hará mayor uso de los servicios de salud y pagará costos más elevados derivados de su atención médica en comparación con personas sin hiperinflación dinámica. El curso temporal de la función respiratoria también provee información valiosa en términos de pronóstico y de gastos en salud.

La fisiología clínica ha evolucionado a la par de los paradigmas científicos. La llamada medicina personalizada o medicina de precisión ha sido un cambio muy importante en el ejercicio clínico. ${ }^{6}$ Por muchos años el modelo osleriano ha sido la mejor forma para practicar la medicina clínica. El modelo osleriano se basa en reconocer patrones para luego otorgarle un nombre y en consecuencia establecer un tratamiento. Por ejemplo, el síntoma A + el síntoma B + ciertos hallazgos funcionales o de imagen forman un patrón «reconocible» (fenotipo) al que le llamamos, digamos, «asma» y de ahí se deriva un tratamiento. Ese exitoso paradigma ha cambiado. Ahora, el modelo toma en cuenta los factores a los cuales el paciente (no los pacientes con síntomas semejantes) ha estado expuesto -el exposoma- y analiza la interacción de dicho exposoma con el genoma del paciente (epigenética) y la forma en la que se establecen «redes» biológicas y sistemas complejos. En este nuevo modelo resulta necesario incorporar a la fisiología clínica los nodos que conformarían a la enfermedad - ¿o red?- llamada «asma», serían, por ejemplo, genoma, exposoma (tabaquismo, contaminantes, alérgenos), bajo peso al nacer, historia de bronquiolitis, función pulmonar, estado socioeconómico, obesidad, inflamosoma, microbiota, presencia de reflujo gastroesofágico, eosinofilia, respuesta Th2, síntomas, hallazgos de imagen, etc. Cada uno de estos nodos interacciona con los demás de manera diferencial y dinámica, lo que genera el llamado endotipo. Lo anterior podría identificar blancos terapéuticos (o preventivos) específicos para un cierto endotipo y de esa manera personalizar el tratamiento. Ante este cambio de paradigma -de la reacción a la prevención y, por ende, al bienestar- la fisiología clínica es parte esencial. Las características fisiológicas formarían un nodo con enorme peso específico por su gran número de interconexiones bidireccionales. Junto con otros nodos, la fisiología clínica forma un sistema biológico y redes complejas. Ante esa forma reciente de entender la medicina, necesitamos fortalecer la formación del neumólogo en el ámbito de la fisiología aplicada para que sepa alimentar a la red con parámetros funcionales provenientes de estudios confiables y con ello mejorar la habilidad predictiva de -los modelos-.

A pesar de esos y muchos otros ejemplos de la utilidad de la fisiología clínica en el ejercicio de la medicina respiratoria, ésta ha sido relegada en los programas de formación de especialistas en neumología. No es muy importante para las sedes formadoras y resulta poco atractivo para los médicos en formación tratar de entender los -aburridos, para algunos- fenómenos biofísicos de la fisiología respiratoria. Es más atractivo y redituable hipertrofiar áreas como la neumología intervencionista o la terapia intensiva que el complicado y matemático apartado de la fisiología. Algunos de los programas más respetables de formación de neumólogos en México no incluyen formación teórica o práctica en fisiología respiratoria. En el mejor de los casos, la fisiología respiratoria ocupa, cuando mucho $2 \%$ del programa de formación de neumólogos. Lo anterior es desafortunado no sólo por las omisiones generadas por la falta del análisis funcional, sino también porque el pensamiento fisiológico es analítico, metódico, crítico y reflexivo, lo que favorece esa actividad tan olvidada que es pensar. El pensamiento del clínico se fortalece enormemente cuando lo hace desde un punto de vista fisiológico. Si en la práctica de la medicina se suprime el análisis escrupuloso, el juicio clínico y la discusión intelectual dejaría de ser medicina y pasaría a ser una ciencia de escalas, guías, modelos, donde las computadoras llevan una holgada delantera.

Más allá de los sorprendentes alcances de la medicina moderna, la fisiología clínica es esencial en la práctica cotidiana de la medicina respiratoria. Las pruebas de fisiología respiratoria deberían ser al neumólogo lo que el electrocardiógrafo es al cardiólogo. Por ello, debemos fortalecer la formación fisiológica de los residentes de neumología. Me refiero no sólo a fortalecer los aspectos técnicos, sino también a dar solidez al pensamiento fisiológico que le permitirá conocer mejor al paciente y a la enfermedad que lo aqueja. El único riesgo que se corre es tener mejores neumólogos. La historia no me dejará mentir: los grandes médicos son también grandes fisiólogos. No podría, creo, ser de otra manera.

\section{REFERENCIAS}

1. Fowler AJ, Wan YI, Carenzo L, Haines RW. COVID-19 phenotypes and potential Hpharm of conventional treatments: how to prove the hypothesis. Am J Respir Crit Care Med. 2020;202(4):619-621. https:// doi.org/10.1164/rccm.202004-1293le

2. Spriggs EA. John Hutchinson, the inventor of the spirometer-his north country background, life in London, and scientific achievements. Med Hist. 1977;21(4):357-364. https://doi.org/10.1017/s0025727300039004

3. Lee HM, Chung SJ, Lopez VA, Wong ND. Association of FVC and total mortality in US adults with metabolic syndrome and diabetes. Chest. 2009;136(1):171-176. https://doi.org/10.1378/chest.08-1901 
4. Celli BR, Cote CG, Marin JM, Casanova C, Montes de Oca M, Mendez $\mathrm{RA}$, et al. The body-mass index, airflow obstruction, dyspnea, and exercise capacity index in chronic obstructive pulmonary disease. N Engl J Med. 2004;350(10):1005-1012. https://doi.org/10.1056/ nejmoa021322

5. Sullivan CE, Issa FG, Berthon-Jones M, Eves L. Reversal of obstructive sleep apnoea by continuous positive airway pressure applied through the nares. Lancet. 1981;1(8225):862-865. https://doi. org/10.1016/s0140-6736(81)92140-1

6. Torre-Bouscoulet L. Los retos de la medicina personalizada. Neumol Cir Torax. 2015;74(4):238-239.

Conflicto de intereses: El autor declara no tener conflicto de intereses. 